\title{
New Beam Position Monitor System Design for the APS Injector*
}

\author{
R. Lill, O. Singh, N. Arnold \\ Advanced Photon Source, Argonne National Laboratory \\ 9700 South Cass Avenue, Argonne, Illinois 60439 USA
}

\begin{abstract}
Demands on the APS injector have evolved over the last few years to the point that an upgrade to the existing beam position monitor (BPM) electronics is required. The injector is presently being used as a source for both the low-energy undulator test line (LEUTL) project and the top-up mode of operation. These new requirements and the fact that many new rf receiver components are available at reasonable cost make this upgrade very desirable at this time. The receiver topology selected is a logarithmic processor, which is designed around the Analog Devices AD8313 log amplifier demodulation chip. This receiver will become the universal replacement for all injector applications measuring positions signals from 352 to $2856 \mathrm{MHz}$ with minimum changes in hardware and without the use of a downconverter. The receiver design features integrated front-end gain and built-in self test. The data acquisition being considered at this time is a $100-\mathrm{MHz}, 12$-bit transient recorder digitizer. The latest experimental and commissioning data and results will be presented.
\end{abstract}

\section{INTRODUCTION}

The Advanced Photon Source injector has taken on a more demanding role over the last few years. The new demands involve operating in top-up mode [1]. In this mode of operation the injector refills the storage ring every two minutes in order to maintain a current of $102 \pm 1.0 \mathrm{~mA}$. In order to inject efficiently into the targeted storage ring bucket, the injector beam position becomes critical. The new beam position monitor (BPM) will provide improved dynamic range, resolution, and reduction of other systematic errors to ensure reliable injector operation.

The low-energy undulator test line (LEUTL) project [2] has also created a new set of demands on the injector. The Linac and several transport lines are shared by both the injection process and LEUTL, requiring new and improved diagnostic capability. These new requirements and the fact that many new rf receiver components are available at reasonable cost make this upgrade very desirable at this time. 


\section{SYSTEM DESIGN}

The system design philosophy was to simplify the topology so the same basic design can be used as the universal replacement for all injector position monitoring applications. The other general design constraint was to make it as reliable and maintainable as possible. The design choice was a log-ratio system with the subtraction function being done in software. The basic expression for the log-ratio beam position $[3,4]$ is:

$$
\begin{aligned}
& N p=\left[(\log (A)-\log (B)]=\log (A / B)=\tanh ^{-1}\left[\frac{(A-B)}{(A+B)}\right]\right. \\
& \approx 2 \frac{A-B}{A+B},
\end{aligned}
$$

where $\mathrm{Np}$ is the normalized position and $\mathrm{A}$ and $\mathrm{B}$ is the induced voltages on the stripline pick-up. This technique adds some flexibility to the system design. The algorithm to calculate the beam position can be changed or adjusted in the future as new techniques develop.

The BPM system was partitioned into subsystems as shown in Figure 1. The stripline detector, bandpass filters, and front-end board make up the major differences between applications. This partitioning allows the use of most of the components for operation at either $352 \mathrm{MHz}$ or $2856 \mathrm{MHz}$. The stripline sensitivity and system calibration factor can be calculated by the following equations $[3,4]$ :

$$
S \approx \frac{80}{\ln (10)} \times \frac{1}{b},
$$

where $\mathrm{S}=$ stripline sensitivity $(\mathrm{dB} / \mathrm{mm})$ and $\mathrm{b}=$ half aperture $(\mathrm{mm})$, and

$$
X(m m) \approx \frac{1}{S \times G_{\text {system }}} \times V_{\text {out }},
$$

where $X(\mathrm{~mm})=$ normalized position $(\mathrm{mm}), G_{\text {system }}=$ system gain (which is set to $36 \mathrm{mV} / \mathrm{dB})$, and $V_{\text {out }}=\log$ amplifier module output $(\mathrm{mV})$.

Table 1 describes the location and number of BPMs that will be upgraded. These applications are transport lines between the linac and the storage ring. This table also describes the type of detector, sensitivity, and system calibration factor. 


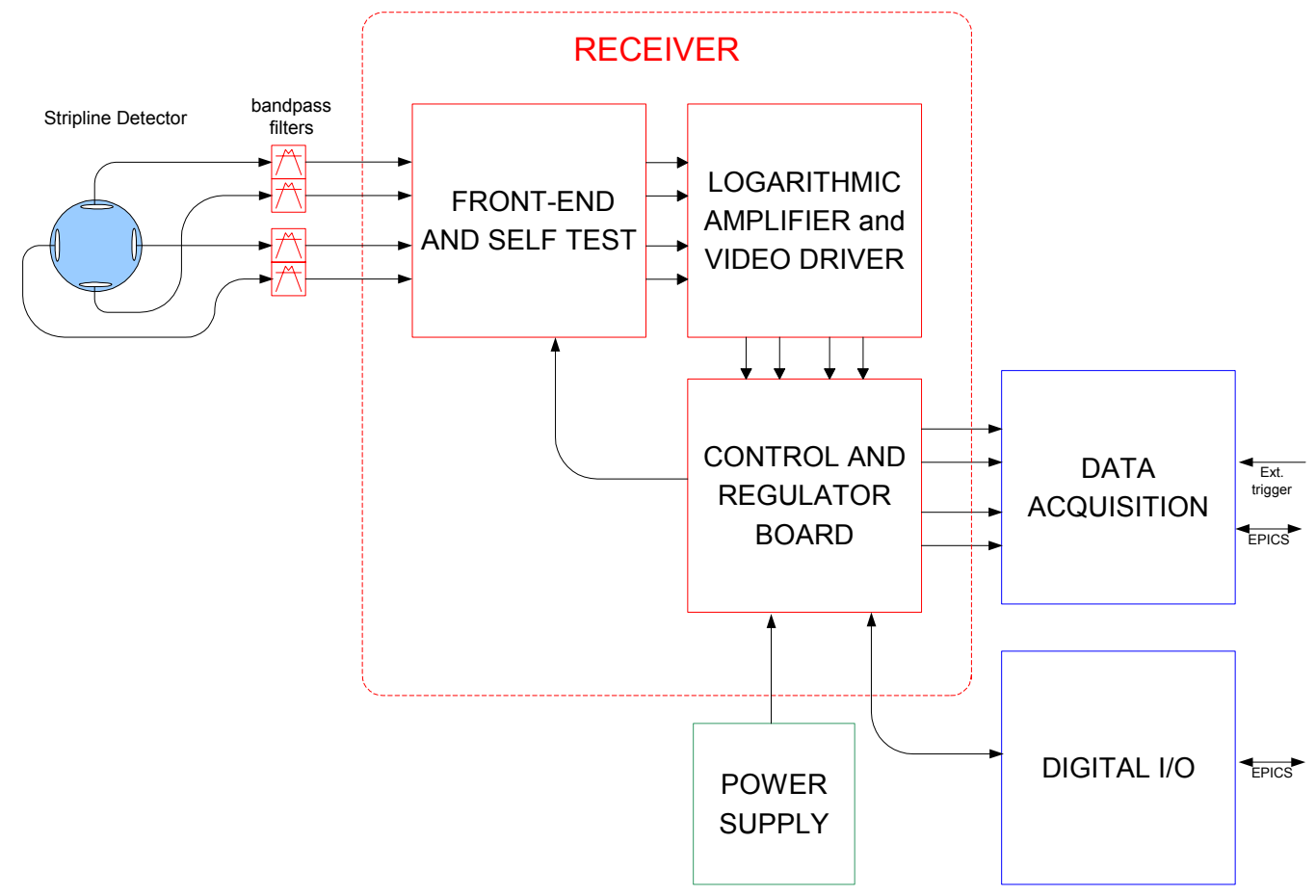

FIGURE 1. System block diagram.

TABLE 1. System Applications.

\begin{tabular}{lccccc}
\hline Location & $\begin{array}{c}\text { Number of } \\
\text { BPMs }\end{array}$ & $\begin{array}{c}\text { Frequency } \\
(\mathbf{M H z})\end{array}$ & $\begin{array}{c}\text { Half } \\
\text { Aperture } \\
(\mathbf{m m})\end{array}$ & $\begin{array}{c}\text { Stripline } \\
\text { Sensitivity } \\
(\mathbf{d B} / \mathbf{m m})\end{array}$ & $\begin{array}{c}\text { Normalized } \\
\text { Position } \\
(\mu \mathbf{m} / \mathbf{m V})\end{array}$ \\
\hline Linac & 15 & 2856 & 17 & 2.0 & $13.6 \times V_{\text {out }}$ \\
LEUTL & 20 & 2856 & $\begin{array}{c}17 \text { and button } \\
\text { type }\end{array}$ & 2.0 & $13.6 \times V_{\text {out }}$ \\
Linac to PAR & 4 & 2856 & 17 & 2.0 & $13.6 \times V_{\text {out }}$ \\
PAR to Booster & 5 & 352 & 25 & 1.4 & $20.0 \times V_{\text {out }}$ \\
$\begin{array}{l}\text { Booster to } \\
\text { Storage Ring }\end{array}$ & 8 & 352 & 25 & 1.4 & $20.0 \times V_{\text {out }}$ \\
\hline
\end{tabular}




\section{RECEIVER DESIGN}

The receiver is partitioned as shown in Figure 1. There is a front-end board, four log amplifier boards, and a control and regulation board for each receiver. The boards are housed in an EMI-shielded aluminum case. The receivers are installed in a 19inch-wide, 4-U height card crate where up to eight receivers can be installed.

\section{Front-End and Self Test Board}

The rf front-end board provides the gain and self-test capabilities for the system. The four signals from the input bandpass filters are sampled via 15-dB directional couplers, which are printed on the circuit board. This provides the ability to troubleshoot the system without disconnecting any cables. The directional couplers also serve as feeds for the self-test oscillators. In the self-test mode the coupler is switched from a $50-\Omega$ termination to a voltage-controlled oscillator. The oscillator drives a two-way equal power divider that is also printed on the circuit board and provides an equal input to one plane of the BPM. This same layout is duplicated for each plane. The board employs a selectable gain stage to shift the operating range by $20 \mathrm{~dB}$. Presently we are using a low-noise Stanford Microdevices SGA-3586 cascadeable gain block with a noise figure of $2.5 \mathrm{~dB}$. There are other amplifiers in this series with different gain and noise parameters that could be implemented in the future. This amplification will shift the input operating range while maintaining the same system gain $\left(G_{\text {system }}\right)$.

This feature will be used in some applications and has the effect of extending the dynamic range.

The front-end and self-test rf board was designed using microstrip transmission lines and components laid out on Rogers RO3006 microwave board materials. The impedance was calculated using Eq. (4) and was optimized in the lab [5]. The dielectric constant of the ceramic PTFE composite material is 6.15 and the loss tangent is $0.0025 @ 10 \mathrm{GHz}$. One of the design goals was keeping the rf board construction process as simple as possible by avoiding bonding substrates. This equates to a two-layer board with a thickness of 0.025 inch to insure the trace width of 0.036 inch for $50-\Omega$ lines. The boards are designed to be drop-in replacements for 352- or 2856-MHz applications.

$$
Z \approx \frac{377}{2 \pi\left(\frac{\left(\varepsilon_{r}+1\right)}{2}\right)^{1 / 2}} \times\left[\ln \left(\frac{8 h}{W}\right)+\frac{1}{8}\left(\frac{W}{2 h}\right)^{2}-\frac{1}{2} \frac{\varepsilon_{r}-1}{\varepsilon_{r}+1}\left(\ln \frac{\pi}{2}+\frac{1}{\varepsilon_{r}} \ln \frac{4}{\pi}\right)\right]
$$

where $\mathrm{Z}=$ microstrip impedance, $\varepsilon_{r}=$ substrate dielectric constant, $\mathrm{h}=$ substrate thickness, and $\mathrm{W}=$ microstrip width. 


\section{Logarithmic Amplifier and Video Driver Board}

The block diagram in Figure 2 shows one of the four logarithmic amplifier video driver boards. The input broadband matching network enables us to use the same hardware for both the 352- and 2856-MHz applications. The AD8313 is one of the main motivations for upgrading the existing 2856-MHz BPMs that require downconverters. The AD8313 uses a cascade of eight amplifier/limiter cells, each having a gain of $8 \mathrm{~dB}$ typically and a $-3 \mathrm{~dB}$ bandwidth of $3.5 \mathrm{GHz}$, for a total gain of $64 \mathrm{~dB}$.

The ADC video driver is the AD8002 dual current feedback amplifier that provides offset and gain adjustment to the log amplifier. In order to evaluate an off-the-self ADC board, the output was designed to drive a $0-2$ volt range with a $1-\mathrm{M} \Omega$ load impedance. The board is a two-layer microstrip design with a thickness of 0.025 inch to insure the trace width of 0.036 inch for $50-\Omega$ lines.

The $\log$ linearity plot shown in Figure 3 illustrates that each gain range has a dynamic rage of about $60 \mathrm{~dB}$ with an overlap of $40 \mathrm{~dB}$. The combination of both ranges enables operation over an 80 - $\mathrm{dB}$ dynamic range with a maximum of $\pm 0.6 \mathrm{~dB}$ errors in $\log$ linearity for $352-\mathrm{MHz}$ applications. The S-band application is expected to be similar with some roll-off at the band edges. The overall pulse rise time for 10 to $90 \%$ of the input is $45 \mathrm{~ns}$ without the lowpass filter implemented for the overall system.

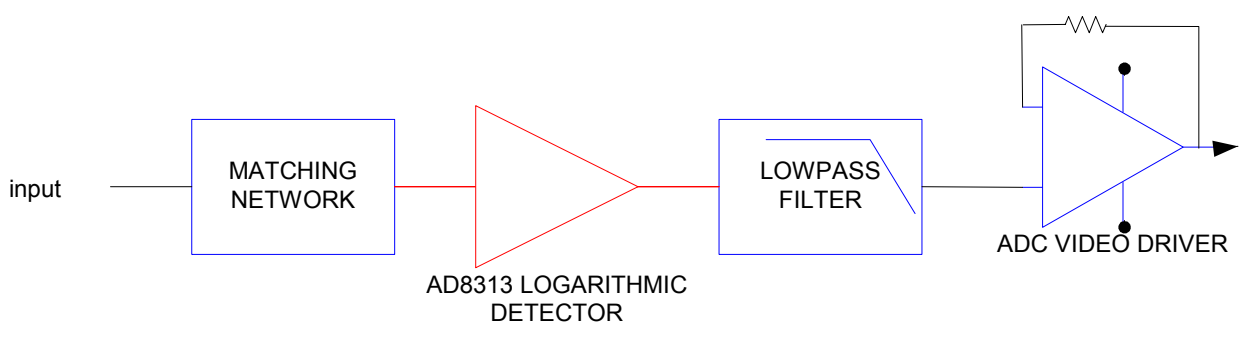

FIGURE 2. One channel log amplifier block diagram.

\section{Control and Regulator Board}

The control and regulator board supplies and distributes regulated power to the five boards in the receiver. It also provides the common point for the control interface. The board employs a hybrid coaxial connector that allows a blind mate connection to the main frame. 


\section{Log Conformance vs. Input Amplitude}

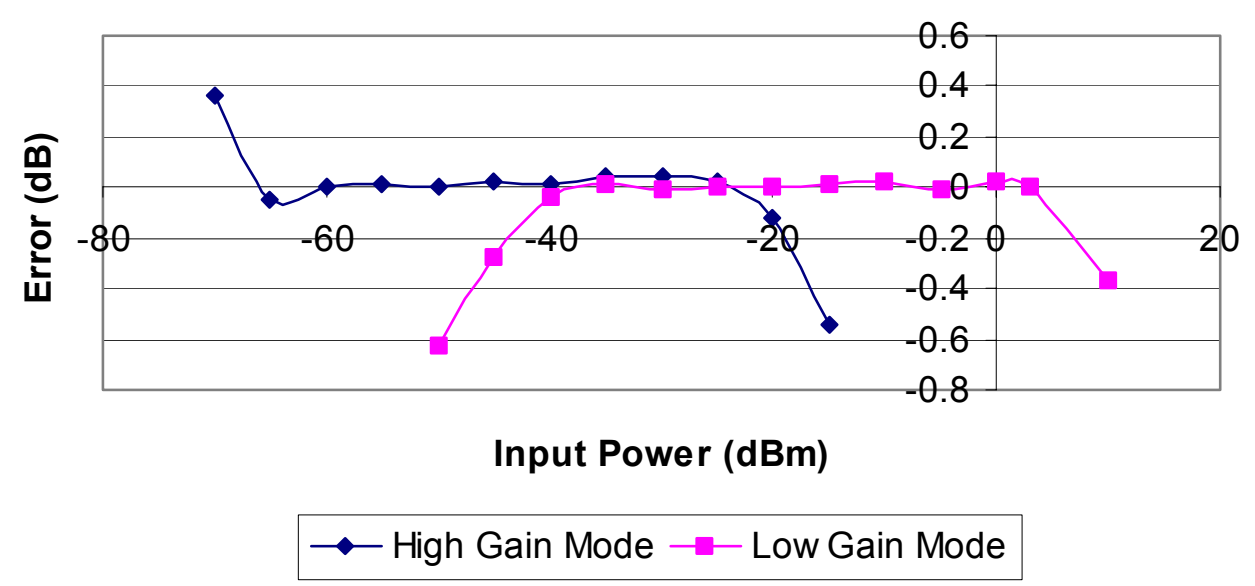

FIGURE 3. 352-MHz receiver logarithmic error.

\section{Striplines and Bandpass Filters}

The 2856-MHz BPMs employ shorted S-band $\lambda / 4$ stripline pickup electrodes, which were recently installed [6], and have a 34-mm-diameter vacuum chamber aperture. The bandpass filters being considered for this application are three-pole $\lambda / 4$ combline filters. The $3-\mathrm{dB}$ bandwidth is $3 \mathrm{MHz}$ with an in-band insertion loss of 6.5 $\mathrm{dB}$.

The 352-MHz BPM applications also utilize a shorted $\lambda / 4$ stripline detector with a vacuum chamber aperture size of $50 \mathrm{~mm}$. They differ from the S-band design not only in blade length but also in width (1.4 rads). The system input bandwidth is limited to a 3-dB bandwidth of 3.0 MHz by Surface Acoustic Wave (SAW) bandpass filters. The SAW filters have matching networks on the input and output with an in-band insertion loss of $7.5 \mathrm{~dB}$. The filter sets are matched to $<0.2 \mathrm{~dB}$ across the $3-\mathrm{MHz}$ band centered at $350 \mathrm{MHz}$. The SAW filter is a very economical ( $\$ 7 /$ filter) solution since it is a standard RFM product.

\section{DATA ACQUISITION}

We are presently evaluating several options for data acquisition at this time. One option is a 12-bit, $100-\mathrm{MHz}$ transient recorder. The VME module contains eight channels, each having $256 \mathrm{~K}$ of memory. Most of the data quoted in this paper was recorded with the 12-bit, $100-\mathrm{MHz}$ digitizer. The other option is a $14-\mathrm{bit}, 50-\mathrm{MHz}$ digitizer. The general approach for using the transient digitizer is to reduce the error or standard deviation by $\frac{1}{\sqrt{N}}$, where $\mathrm{N}$ is the number of samples collected during the 
video pulse. In the $352-\mathrm{MHz}$ application we can take as many as 30 samples or $300 \mathrm{~ns}$ from the start of the event.

\section{PERFORMANCE}

We have built and are currently testing prototypes for a $352-\mathrm{MHz}$ receiver and a 2856-MHz receiver at this time. The plot shown in Figure 4 is the output of the 352$\mathrm{MHz}$ system operating with the 12-bit, $100-\mathrm{MHz}$ digitizer. The input to the BPM system is a simulated beam pulse with the data acquisition set to acquire 30 samples from a single pulse. The top trace is an overlay of all four-blade signals as a function of input power. The second trace shows the position intensity dependence with error bars representing the noise. The third trace shows the peak-to-peak noise of each of the four blades as a function of input power.

The 352-MHz system was also tested in the lab with a $50-\mathrm{mm}$ stripline chamber installed in a wire test set-up. The wire test accuracy was $19 \mu \mathrm{m}$ rms over a $\pm 5 \mathrm{~mm}$ range and $8 \mu \mathrm{m}$ rms over a $\pm 1 \mathrm{~mm}$ range. The lab testing indicates that the overall electronic system drift stability is $<15 \mu \mathrm{m}$ over a 48-hour period.

The 352-MHz system testing is currently moving from the lab to the accelerator for further evaluation. The single-shot resolution for this system installed in booster-tostorage ring (BTS) transport line is $15 \mu \mathrm{m}$ over a 30-dB dynamic range. Further testing will continue after the May shut down.
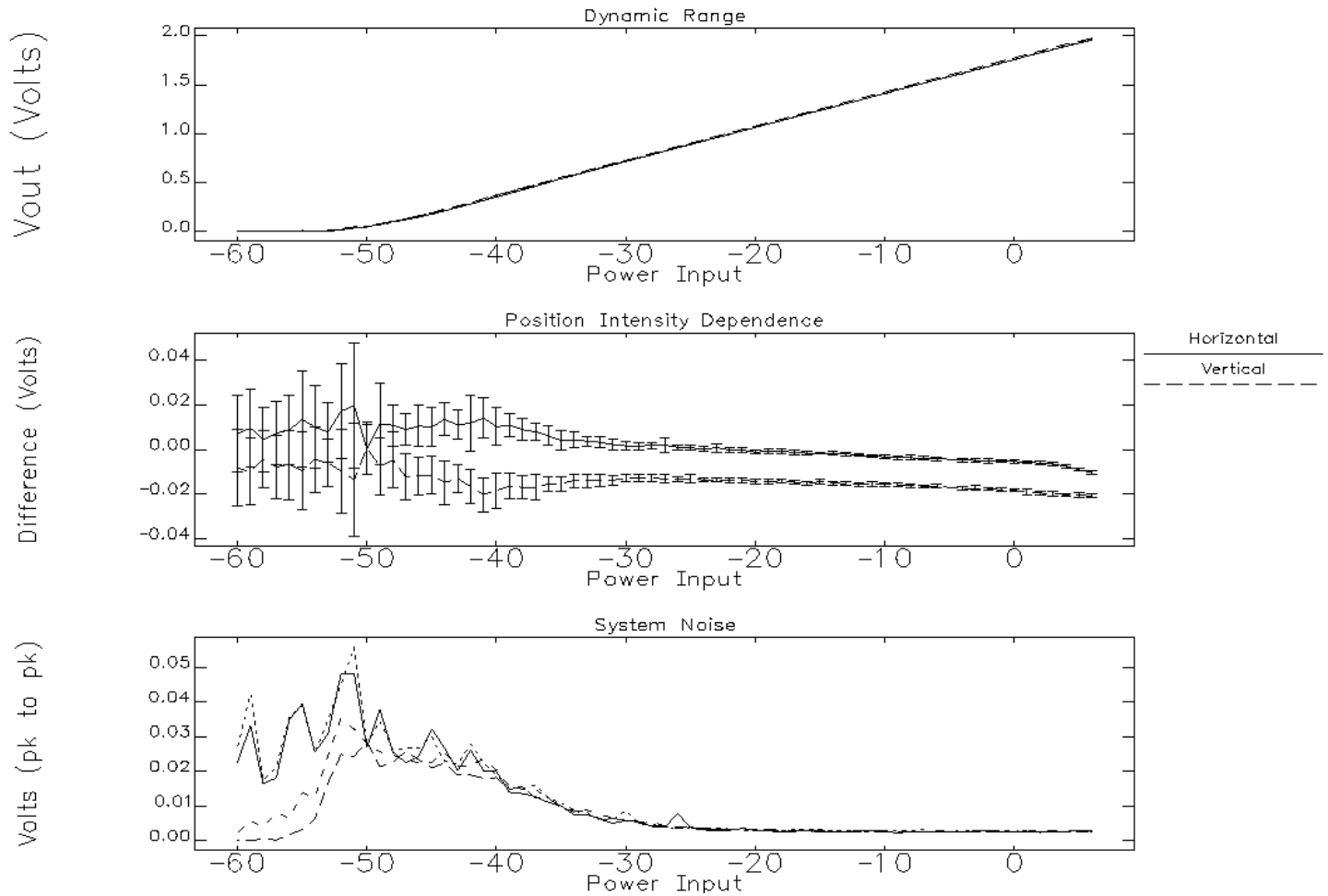

FIGURE 4. 352-MHz performance. 
TABLE 2. Design Specifications.

\begin{tabular}{lcc}
\hline Parameter & PC Gun & RF Gun \\
\hline Dynamic Range & $0.1-2 \mathrm{nC}(26 \mathrm{~dB})$ & $0.1-10 \mathrm{nC}(40 \mathrm{~dB})$ \\
Single Shot Resolution & $15 \mu \mathrm{m} \mathrm{rms}$ & $100 \mu \mathrm{m} \mathrm{rms}$ \\
Drift & $15 \mu \mathrm{m} \mathrm{rms}$ & $100 \mu \mathrm{m} \mathrm{rms}$ \\
Accuracy & $100 \mu \mathrm{m} \pm 5 \mathrm{~mm}$ & $100 \mu \mathrm{m} \pm 5 \mathrm{~mm}$ \\
\hline
\end{tabular}

\section{DISCUSSION}

The BPM system was designed to meet the parameters given in Table 2. The 352$\mathrm{MHz}$ system satisfies the given requirements; however some enhancements warrant investigation. We will explore reducing the single-shot noise by implementing a differentially driven log amplifier output. This will reduce the common mode broadband noise.

The S-band receiver will be more challenging. However, if the signal-to-noise ratio remains relatively constant, we should see about a 32\% improvement in the resolution due to the fact that the chamber aperture is smaller.

The plan is to complete testing on the two prototypes by mid-summer, followed by ramping-up production of the first-article units by late summer.

\section{ACKNOWLEDGMENTS}

The author would like to acknowledge Glenn Decker for many helpful discussions, Tom Buffington for the mechanical design work, and Robert Keane and Chuck Gold for providing valuable electrical measurement and assembly expertise.

\section{REFERENCES}

1. Emery, L., "Recent Operational Data on Continuous Top-Up Operation at the Advanced Photon Source," PAC 2001 Conference Proceedings, Chicago, pp. 2599-2601 (2001).

2. Milton, S., "Measurements of Exponential Gain and Saturation of SASE at the APS LEUTL," PAC 2001 Conference Proceedings, Chicago, pp. 236-240 (2001).

3. Shafer, R., "Beam Position Monitoring," AIP Conference Proceedings No. 212, Upton, NY. pp. 2658 (1989).

4. Aiello, G. and Mills M., "Log-Ratio Technique For Beam Position Monitor Systems," AIP Conference Proceedings No. 281, Berkeley, CA, pp. 301-310 (1992).

5. Gupta, K. C. et. al., Microstrip Lines and Slotlines, Artech House Publishers, Boston, London 1996.

6. Gorski, A. and Lill, R., "Construction and Measurement Techniques for the APS LEUTL Project RF Beam Position Monitors," Proceedings of the 1999 Particle Accelerator Conference, New York, pp. 1411-1413 (1999). 